\title{
Application of Data Mining Technology in the Early Warning of Safety Risks
}

\author{
Wenxuan Yang \\ Gansu Natural Resources Information Center, Information \\ Center, Gansu, China
}

\begin{abstract}
With the advent of the Internet era, Internet, cloud computing, and big data technologies are developing rapidly and their applications are becoming more mature. In this context, it is essential to pay attention to the construction of network system and information safety. At present, there are increasing number of risk events caused by insufficient network defense and early warning. Although many enterprises, administrative departments and individuals pay attention to the hidden dangers of data safety and apply innovative data mining technology to risk prevention, the limitation of the application of data mining technology is also very obvious, most of which guides the rectification through the analysis of hidden danger data, but ignores the mining and utilization of the potential value behind the data. Based on this, this work first analyzed the implementation method and framework of data mining technology in the early warning of safety risk, and then summarized system requirements and safety risk prediction model taking food safety risk as an example.
\end{abstract}

\section{CCS CONCEPTS}

- Computing methodologies $\rightarrow$ Modeling and simulation; Model development and analysis.

\section{KEYWORDS}

Data mining technology, information age, applied research, risk resistance

ACM Reference Format:

Wenxuan Yang and Yi Liu. 2021. Application of Data Mining Technology in the Early Warning of Safety Risks. In 2021 2nd International Conference on Artificial Intelligence and Information Systems (ICAIIS '21), May 28-30, 2021, Chongqing, China. ACM, New York, NY, USA, 5 pages. https://doi.org/10. $1145 / 3469213.3470313$

\section{INTRODUCTION}

Big data is not a novelty, and the wide and mature application of big data technology also promotes the development of big data era. Big data corresponds to massive data information. How to mine truly valuable information from massive and complicated information to realize system integration and optimization analysis of data is important for the subsequent application of big data technology.

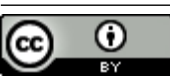

This work is licensed under a Creative Commons Attribution International 4.0 License.

ICAIIS '21, May 28-30, 2021, Chongqing, China

(c) 2021 Copyright held by the owner/author(s)

ACM ISBN 978-1-4503-9020-0/21/05

https://doi.org/10.1145/3469213.3470313

\author{
Yi Liu \\ University of California, Riverside, Dept of ECE, Wuhan, \\ China, yliu726@ucr.edu, Corresponding author
}

Strengthening the mining and analysis of the data value behind big data can identify risks based on the perspective of safety risk prevention, improve users' awareness of risk early warning and attention, and also build safety barriers for information safety. As one of the kinds of big data, the big data of safety production gets insufficient research and attention, since it focuses more on the theory, data source analysis and data methods analysis, but ignores the research on the safety application [1]. With the rapid development of network technology, network safety risk has attracted more and more attention. It is necessary to explore the value of data mining technology in safety risk control and do a good job in the application and analysis of risk early warning.

\section{KEY IMPLEMENTATION METHODS}

\subsection{Overview of BP neural network}

With the rapid development of information technology and computer network, the channels for people to obtain information and the amount of data show explosive growth. How to extract effective information from massive data has become an urgent task. Data mining technology is an information processing technology developed to meet this demand. Based on special algorithms, it extracts effective information from a large number of noisy, incomplete and fuzzy data, and reveals the hidden law, so as to effectively predict and correctly guide future work. At present, the main data mining techniques include neural network, genetic algorithm, rough set, support vector machine and decision-making tree, among which neural network method has strong self-learning and associative storage function, which can search for optimal solutions at high speed, and has a wide range of applications in demand prediction. BP neural network is a multi-layer feed-forward network based on $\mathrm{BP}$ algorithm. Its algorithm is mature, the network structure is simple, and it has outstanding characteristics of strong nonlinear mapping capability [2]. The essence of BP algorithm is to solve the minimum value of error function, and the traditional BP algorithm is a supervised learning algorithm based on the least square method. BP algorithm trains the network through data sample sets of input and output according to the principle of error reverse transmission. The learning process includes the forward transmission process of information and the reverse transmission process of error, which are repeatedly used to continuously calculate the changes of network weights and deviations in the direction of gradient decline of relative error function, and gradually approach the target. A typical BP neural network consists of an input layer, at least one hidden layer and one output layer. A typical three-layer BP neural network structure is shown in Figure 1 


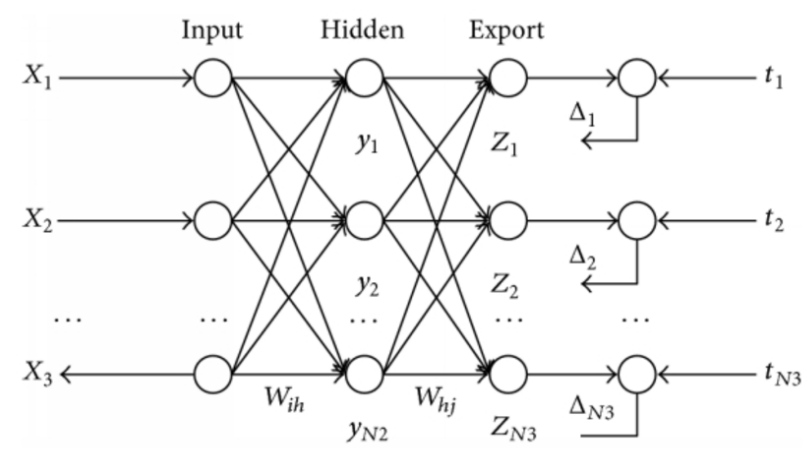

Figure 1: BP Neural Network Structure Diagram

\subsection{BP algorithm}

In BP neural network, let the output be $\mathrm{y}$ and the input corresponding to any node $\mathrm{i}$ be $\mathrm{O}_{\mathrm{i}}$. It is assumed that there are $\mathrm{N}$ samples $\left(\mathrm{x}_{\mathrm{k}}, \mathrm{y}_{\mathrm{k}}\right)(\mathrm{k}=1,2, \ldots \mathrm{N})$, the network input is $\mathrm{x}_{\mathrm{k}}$, the network output is $y_{k}, \mathrm{O}_{\mathrm{ik}}$ represents the output of node $\mathrm{i}$ in the network, and the input of node $\mathrm{j}$ is:

$$
\text { netjk }=\sum_{\mathrm{i}} W \mathrm{ijOij}
$$

The formula of the square error function is:

$$
E=\frac{1}{2} \sum_{\mathrm{k}}^{N}\left(\mathrm{yk}-\mathrm{yk}^{\prime}\right)
$$

Where $\mathrm{y}_{\mathrm{k}}$ ' is the actual network output, which can be defined as:

$$
\begin{gathered}
E \mathrm{k}=\left(\mathrm{yk}-\mathrm{yk}^{\prime}\right)^{2} \\
O \mathrm{jk}=\mathrm{f}(\text { netjk })
\end{gathered}
$$

Where,

$$
\begin{aligned}
\mathrm{f}(\mathrm{x}) & =1 / 1+e^{-x} \\
\delta \mathrm{jk} & =\frac{\partial E \mathrm{k}}{\partial \mathrm{net} \mathrm{k}}
\end{aligned}
$$

Thus it can be derived that:

$$
\frac{\partial E \mathrm{k}}{\partial \mathrm{netij}}=\frac{\partial E \mathrm{k}}{\partial \mathrm{netjk}} * \frac{\partial \mathrm{net} \mathrm{jk}}{\partial \mathrm{netij}}=\delta \mathrm{jkOij}
$$

\section{IMPLEMENTATION FRAMEWORK: EARLY WARNING OF SAFETY RISKS BASED ON DATA MINING}

\subsection{Case introduction}

The risk early warning system of food safety is an important means to improve food safety production supervision and food production level. Under the leadership of the government, many places in China have established relatively perfect early warning system of food safety risk, which can serve the development of market economy. The current early warning system of food safety is mainly aimed at testing object or the project itself and the sampling rate for early warning, but considers other factors relatively little. The main reason is that the regulatory authorities can roughly understand the risk of food through the detection data and sampling frequency of food, so as to issue early warning information, which can realize the early warning of food. However, these warning systems also have some problems. First, the system itself has some shortcomings, such as incomplete raw data and noise, so there is a certain deviation in food safety risk warning, which cannot reflect the real risk level. Second, with the development of economy and the globalization of trade, there are more and more risk factors affecting food safety, including enterprise risk, official risk, food risk, social attention, and inspection standards. For example, different countries have different testing standards for the same testing items. Japan has the lowest requirement for pesticide residues in food testing standards, while domestic national standards have the highest requirement. Therefore, it is not convincing to simply measure food safety with inspection data [3]. Therefore, it is not convincing to simply use test data to measure food safety. For instance, even though the food of some enterprises is qualified, the imminent construction of chemical plants around the enterprise, and the frequency of supervision by regulatory departments is zero in the quarter, and many violations in commodity trade may indirectly lead to the occurrence of food safety problems. Therefore, relying solely on food inspection data as the most important data for food safety warning can no longer meet the needs of the market and can not guarantee food safety more comprehensively. It is necessary to make full use of a large number of valuable historical data accumulated by inspection and quarantine departments, and design a comprehensive risk early warning information system of food safety by integrating the information of departments, enterprises and society [4].

\subsection{System requirement analysis}

The participants of this system mainly have three kinds of personnel: information provider, system administrator and query personnel.

- Information provider

The information provider includes supervision department personnel and enterprise personnel, which are responsible for providing inspection information, inspection standard information, supervision information, enterprise information and enterprise inspection information, as shown in Figure 2. Supervisory department personnel carries out inspection standard, inspection information and supervision information through the login system. In terms of inspection standard input, it is necessary to release, update and delete information according to national standards, and remind users to inquire and use. In terms of input inspection information, the enterprise food inspection data provided by the inspection institutions shall be input in turn. In terms of regulatory information input, supervision frequency, supervision personnel and supervision results can be recorded according to actual situation. This feature provides import capabilities to reduce the workload of the staff.

- System administrator

System administrator is the main user of the system, which is responsible for query, system management, statistics, and early warning of management and maintenance. The structure and function of the system administrator are shown in Figure 3. The query function includes enterprise inspection query, enterprise information query, inspection standards query, food information query and 


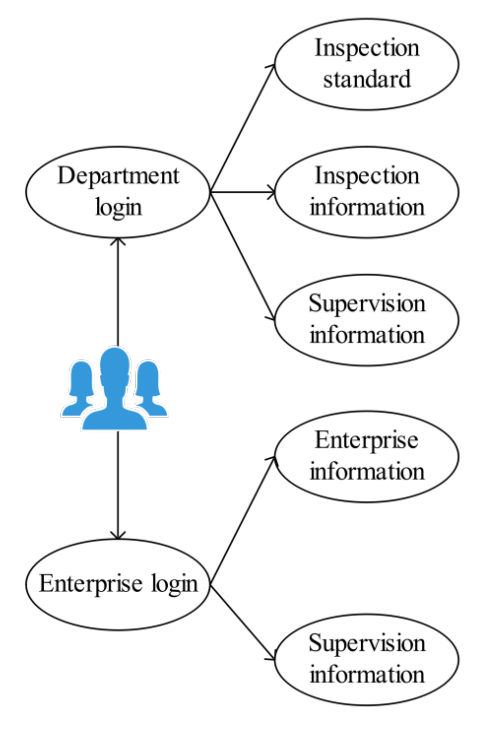

Figure 2: Structure and function of information provider

other sub-functions. Through accurate retrieval and comprehensive retrieval, the enterprise inspection information, enterprise information, inspection standard information and food information can be queried. If the information is not queried, the system administrator can enter the system management process and put forward demands on the information provider, requiring departmental users or enterprise users to re-enter or supplement the information input. The structure and functions of the system inquiry flow chart are shown in Figure 4. System management functions include right management, user maintenance, data maintenance and other subfunctions [5]. The administrator realizes the user authority and user other information management function, as well as the realization of the system related data maintenance function. In this system, there is only one initial user, that is, the system administrator, who can supervisory department users through the system administrator and grant the corresponding authority. Since the permissions of enterprise users are relatively uniform, users of regulatory departments only need to select the default when granting rights to enterprise users. The main authority of department users is to add enterprise users, maintain inspection standard information and so on. The main authority of department users is to add enterprise users and maintain inspection standard information. The main rights of enterprise users include the maintenance of their own basic information and enterprise inspection information. The system management process is shown in Figure 5

\section{- Query personnel}

Users of early warning system can retrieve and view early warning information. The query personnel structure is shown in Figure

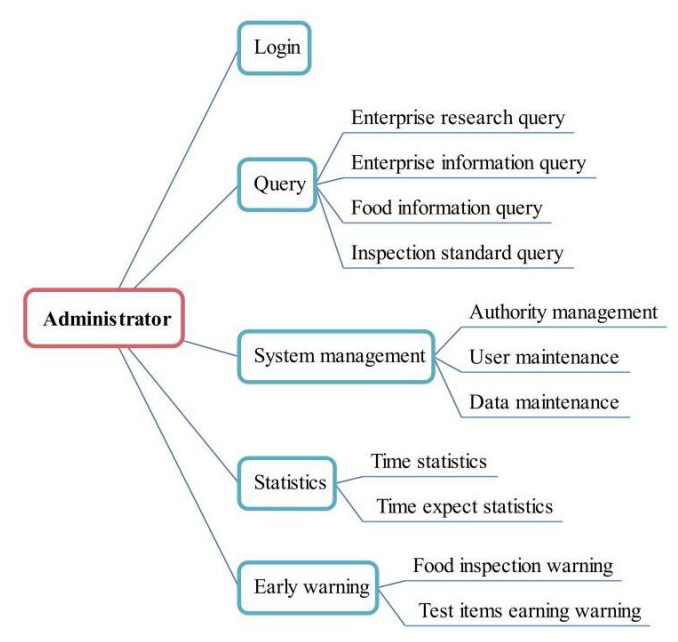

Figure 3: Structure and function of system administrator

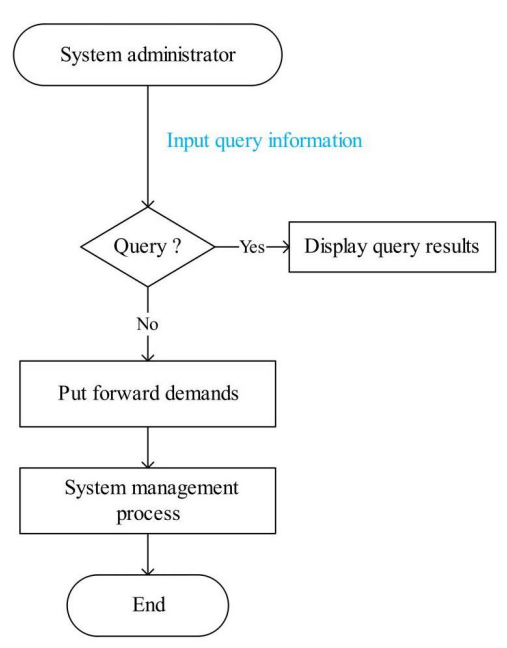

Figure 4: System query flow chart

6. In terms of the early warning information retrieval function, if the inquirer enters the food retrieval information, such as the mooncake of A Company, the system will display the early warning information of the food reported for inspection.

\subsection{Food risk model}

There are many kinds of hazardous substances contained in food, which can be classified according to the category of hazardous substances, and can be divided into the following seven types: pesticide residue, biotoxin, microorganism, additive, non-usable substance, 


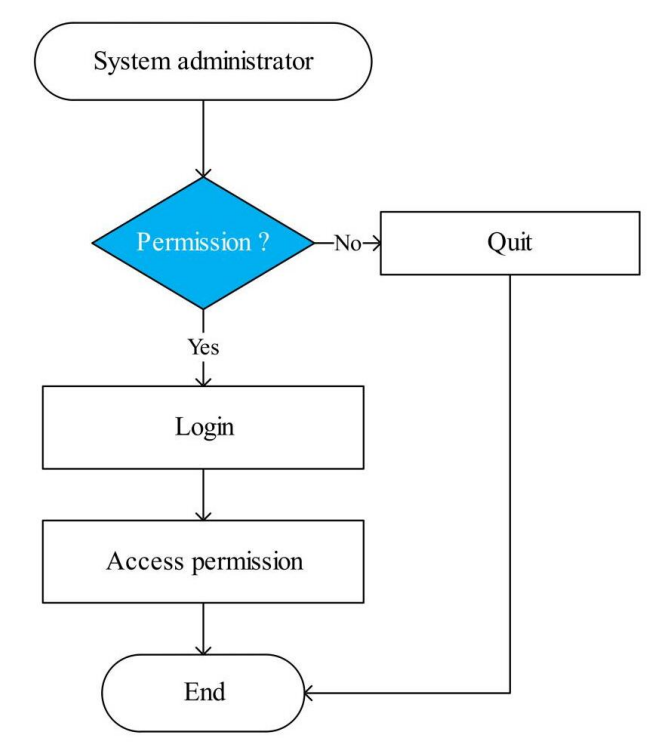

Figure 5: System management flow chart

heavy metal and chemical pollutant. According to the degree of harm, hazardous substances can be classified into restricted use and prohibited use of hazardous substances. If the prohibited hazardous substance is detected in a food product, the risk level of the product is considered very high. Therefore, if the reported data of food testing contains hazardous substances that are prohibited to be used, the food will be classified as high risk food, and there is no need to continue risk analysis. The hazardous substances in different foods are different, so the hazardous substances that need to be tested are also different. For example, the main hazardous substances in dairy products are biotoxins, mainly including salmonella and nitrite, and the main hazardous substances in vegetables are pesticide residues.

According to the analysis of historical data, food risk mainly includes the probability of occurrence of hazards, the range of nonconformity and the social attention. First, when inspecting the food submitted for inspection, if the food contains the hazardous substance and is detected as unqualified, the risk level of the hazardous substance is considered to be high. Second, another important basis for risk assessment of hazards is the range of unqualified data. The unqualified data means that the residual amount of hazards contained in food exceeds the standard value in the safety monitoring of food. In practical work, it is necessary to pay attention to the detection data and find out the reasons for the unqualified data. Third, the response of harm in people's life is the social attention of the harm. Social attention is regarded as a risk factor of harm, mainly because when a harm causes high social attention, the national supervision department will strengthen the detection of the harm [6].

It can be seen that food risk evaluation indicators are complex and diverse, and have hierarchical characteristics. Different indicators are composed of many sub-indicators, and the number and

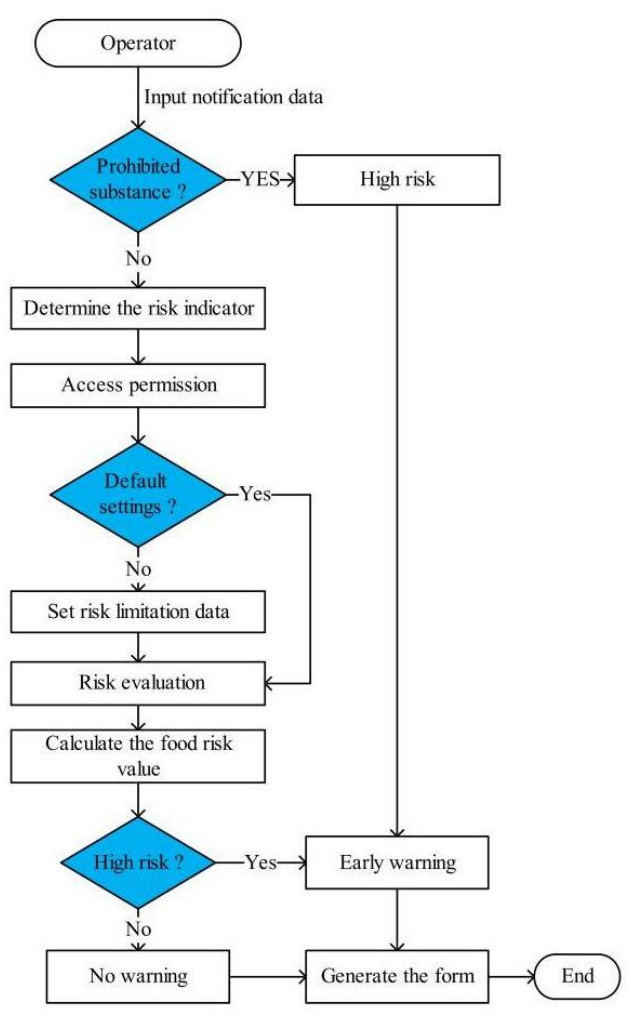

Figure 6: Risk warning of food safety flowchart

content of sub-indicators are different. Therefore, this work adopted hierarchical comprehensive evaluation method to evaluate food risk. According to the index system of food risk evaluation, a three-level evaluation system is constructed. The index factor of the first-level risk evaluation system is food risk, the evaluation results are taken as the input values of the next level risk evaluation system, and the overall food risk evaluation level is obtained by this method. Based on this, the food risk index level and weight setting can be obtained, as shown in Figure 7

\section{CONCLUSION}

At present, the channels of information acquisition are more diversified, and the surge trend of data volume is more obvious. It is a great challenge for people to find the key valuable information from the massive and complicated data. The birth and effective application of data mining technology will bring about the effective screening and integration of data [7]. Data mining technology is used to guide the control of safety risks, especially the control of food safety risks, so as to realize the supervision of the whole food safety industry chain, avoid food risk events, and ensure the stable development of the food industry. It also enhances the public credibility of social food safety, so that people have more confidence in food safety. This work introduced the overall structure design of food safety risk warning system based on data mining, analyzed the realization of risk warning function in detail, and designed the business logic function of the system. 


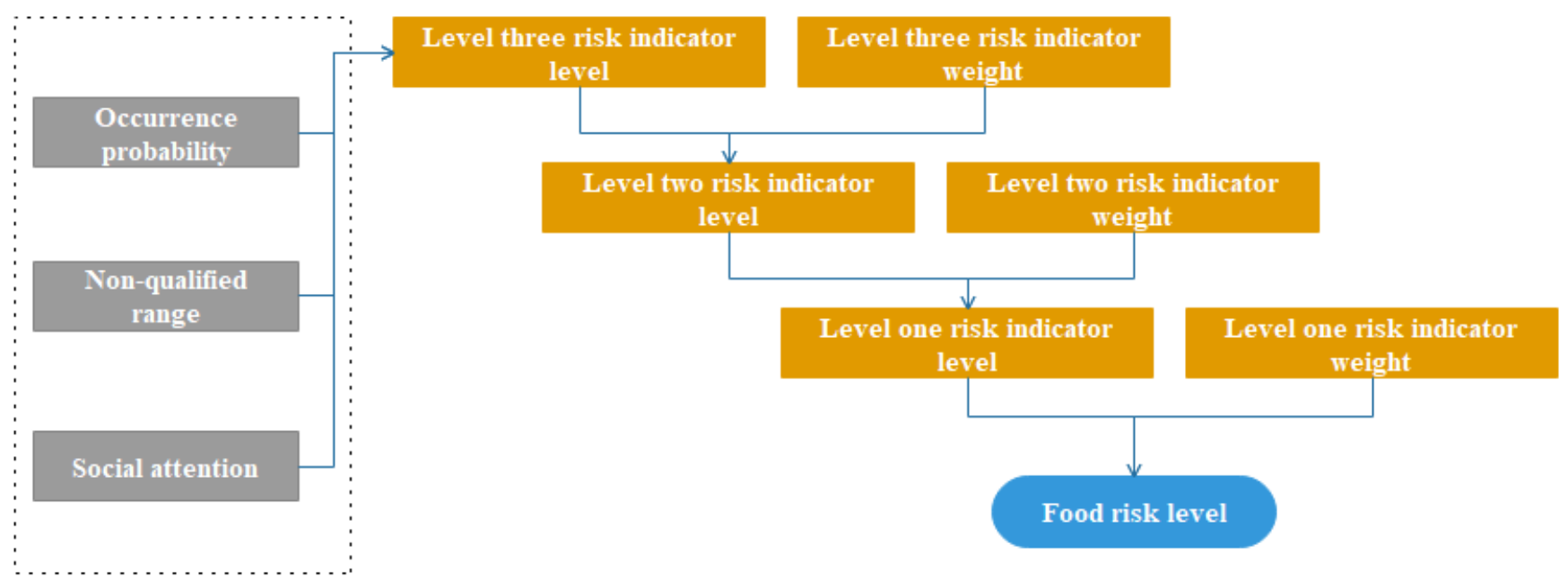

Figure 7: Food risk indicator level and weight setting diagram

\section{REFERENCES}

[1] Chong-mao, L., Rui, N., \& Xiang-yan, Q. (2015). Forecast and Prewarning of Coal Mining Safety Risks Based on the Internet of Things Technology and the Big Data Technology.

[2] Moradpour, S., \& Long, S. (2019). Using Combined Multi-Criteria Decision-Making and Data Mining Methods for Work Zone Safety: A Case Analysis. Case studies on transport policy, 7, 178-184.

[3] Viloria, A., Lopez, J.R., Llinas, N.O., Mercado, C., Coronado, L.E., Sepúlveda, A.M., \& Lezama, O.B. (2020). Prediction of Psychosocial Risks in Teachers Using Data Mining.
[4] Wang, J., \& Yue, H. (2017). Food safety pre-warning system based on data mining for a sustainable food supply chain. Food Control, 73, 223-229.

[5] Tubert-Bitter, P., Bégaud, B., \& Ahmed, I. (2016). Comparison of two drug safety signals in a pharmacovigilance data mining framework. Statistical Methods in Medical Research, 25, 615 - 629 .

[6] Kajungu, D.K., Erhart, A., Talisuna, A., Bassat, Q., Karema, C., Nabasumba, C., Nambozi, M., Tinto, H., Kremsner, P., Meremikwu, M., d'Alessandro, U., \& Speybroeck, N. (2014). Paediatric Pharmacovigilance: Use of Pharmacovigilance Data Mining Algorithms for Signal Detection in a Safety Dataset of a Paediatric Clinical Study Conducted in Seven African Countries. PLoS ONE, 9.

[7] Goren, A. (2013). A Gold Mine of Information: Using Pharmaceutical Data Mining to Ensure Long-term Safety and Effectiveness of Pharmaceuticals. 